\title{
Screening and functional analyses of Nilaparvata lugens salivary proteome
}

Hai-Jian Huang, Cheng-Wen Liu, Xiao-Hui Huang, Xiang Zhou, Ji-Chong Zhuo, Chuan-Xi Zhang, Yan-Yuan Bao*

State Key Laboratory of Rice Biology and Ministry of Agriculture Key Laboratory of Agricultural Entomology, Institute of Insect Sciences, Zhejiang University, Hangzhou 310058, China

* Corresponding author. Tel: +86-571-88982995; Fax: +86-571-88982991

E-mail address: yybao@zju.edu.cn (Y.-Y. Bao)

E-mail addresses for all authors

Yan-Yuan Bao: yybao@zju.edu.cn

Hai-Jian Huang: huanghaijian@zju.edu.cn

Cheng-Wen Liu: 21416113@zju.edu.cn

Xiao-Hui Huang: 21316178@zju.edu.cn

Xiang Zhou: xiangzhou @zju.edu.cn

Ji-Chong Zhuo:11316091@zju.edu.cn

Chuan-Xi Zhang: chxzhang@zju.edu.cn

Supporting Information, table of contents

Figure S1: Detection of watery and gelling saliva protein components by SDS-PAGE.

Figure S2: Determination of gene expression changes after RNAi

Figure S3: Annotated tandem mass spectra of five watery saliva proteins and five gelling saliva proteins

Table S1: Primers used in quantitative real-time PCR and double stranded RNA synthesis 


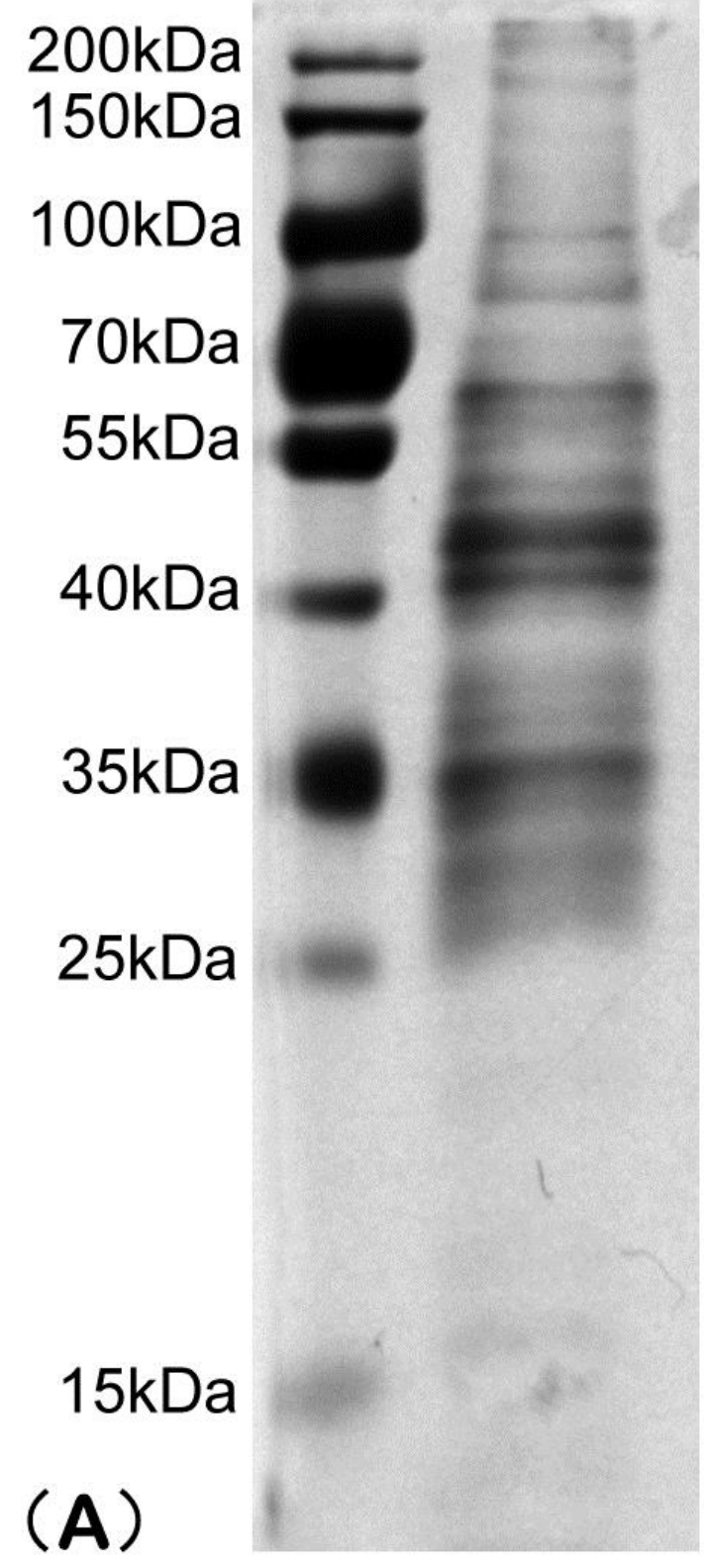

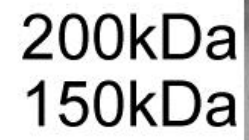

100kDa

$70 \mathrm{kDa}$

$55 \mathrm{kDa}$

$40 \mathrm{kDa}$

$35 \mathrm{kDa}$

$25 \mathrm{kDa}$

$15 \mathrm{kDa}$

(B)

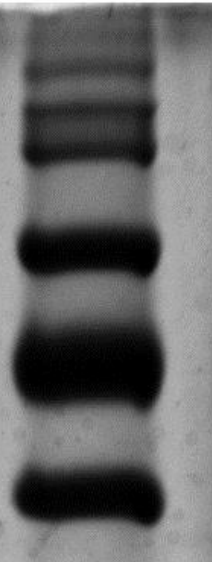

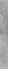

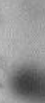




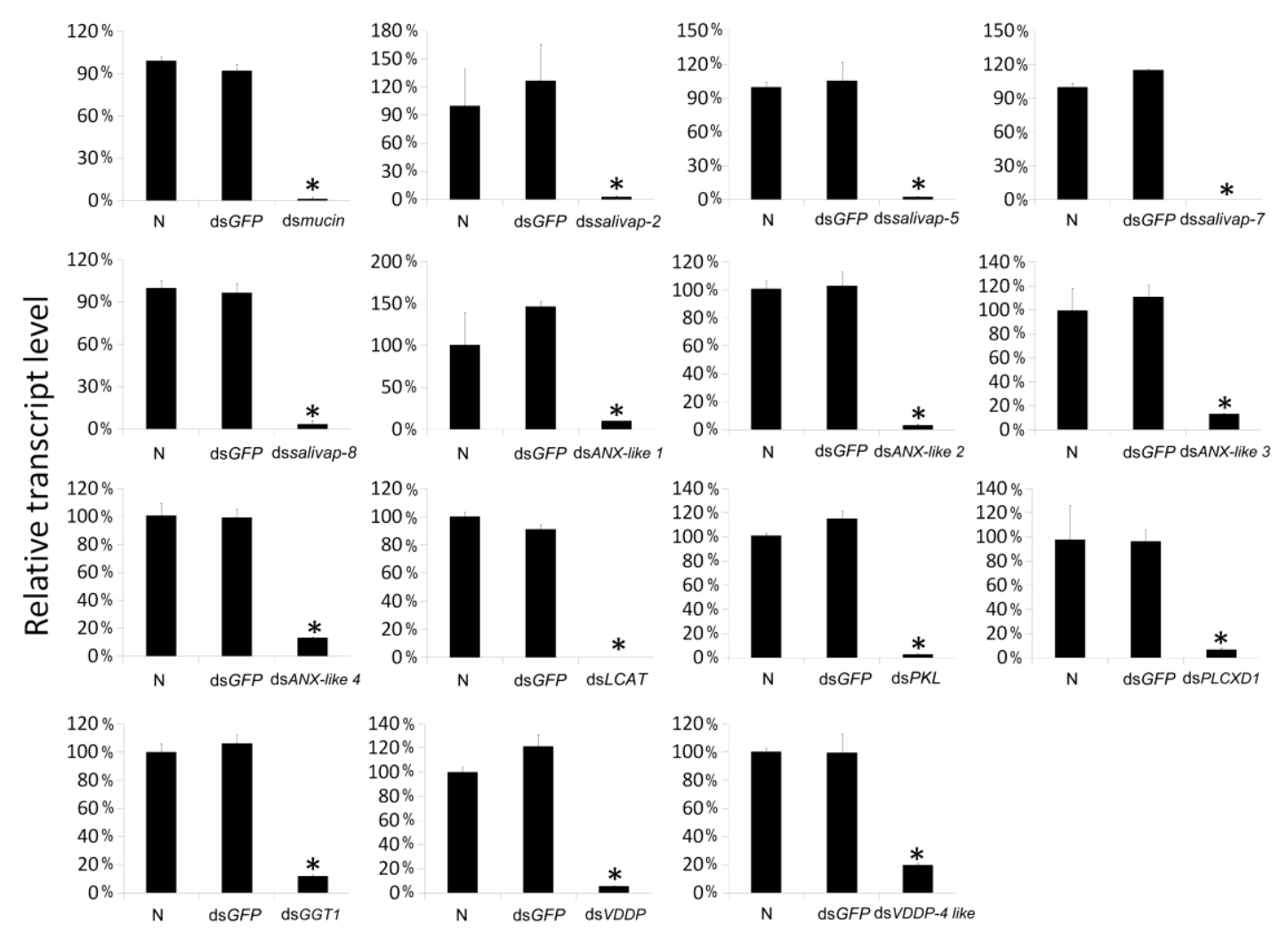

Figure S2. Determination of gene expression changes after RNAi. The third instar nymphs were injected with specific dsRNA. Individuals treated with dsGFP and untreated were used as controls. The gene expression variations (in \%) at $5 \mathrm{~d}$ p.i. in $N$. lugens were analyzed by quantitative real-time PCR as described in Figure 3. 
Annotated tandem mass spectra of five watery saliva proteins of $N$. lugens
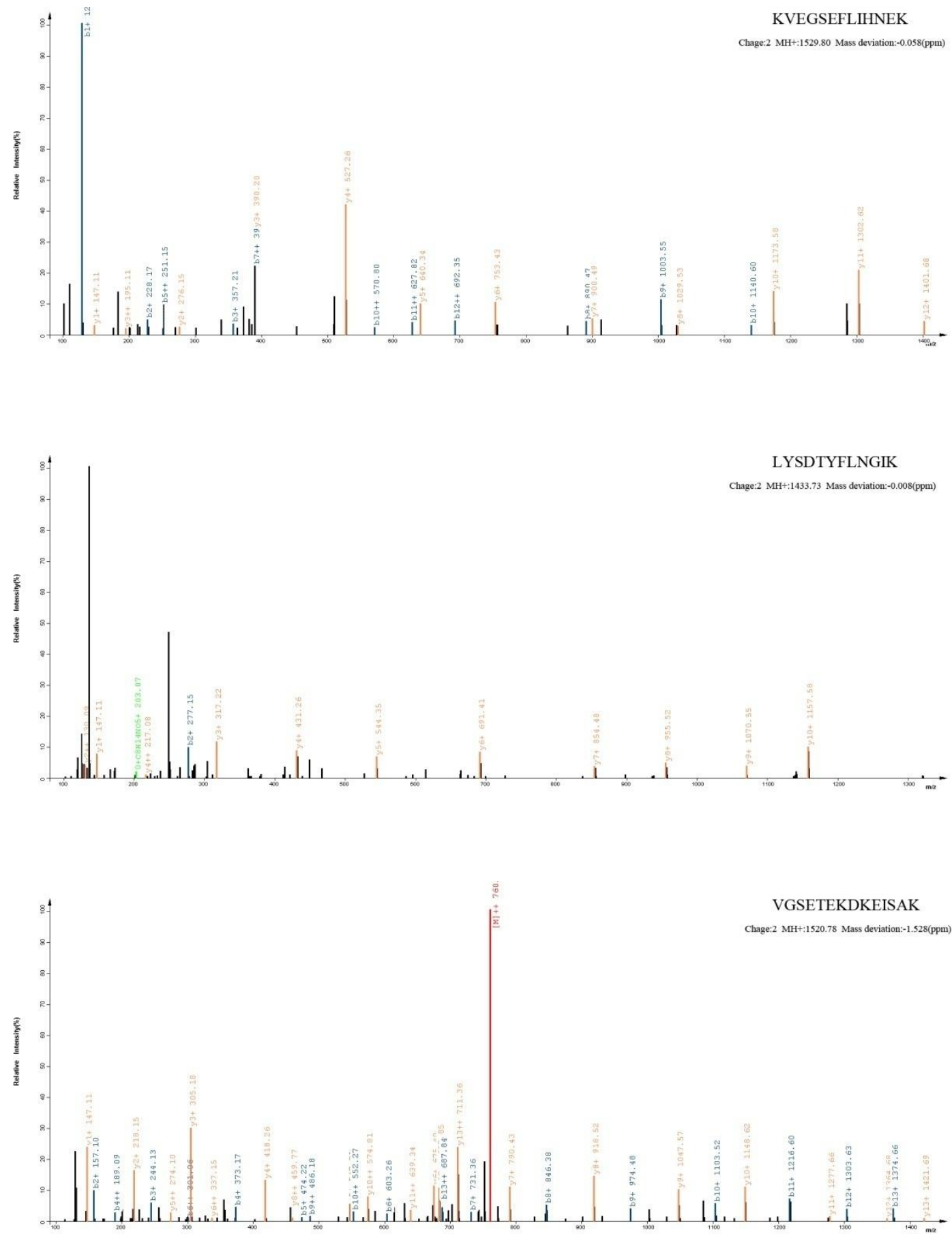

carboxylesterase 

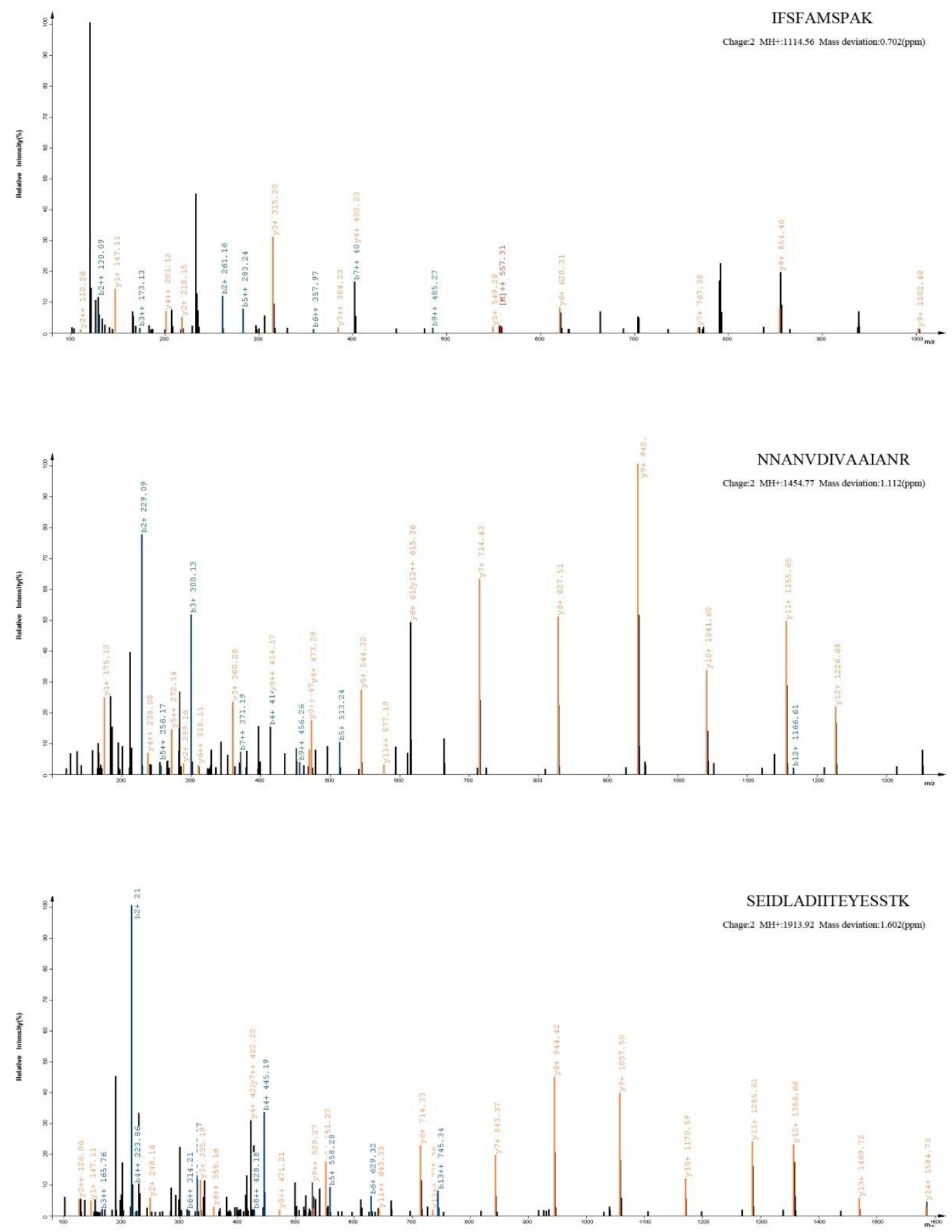

ANX-like 1 

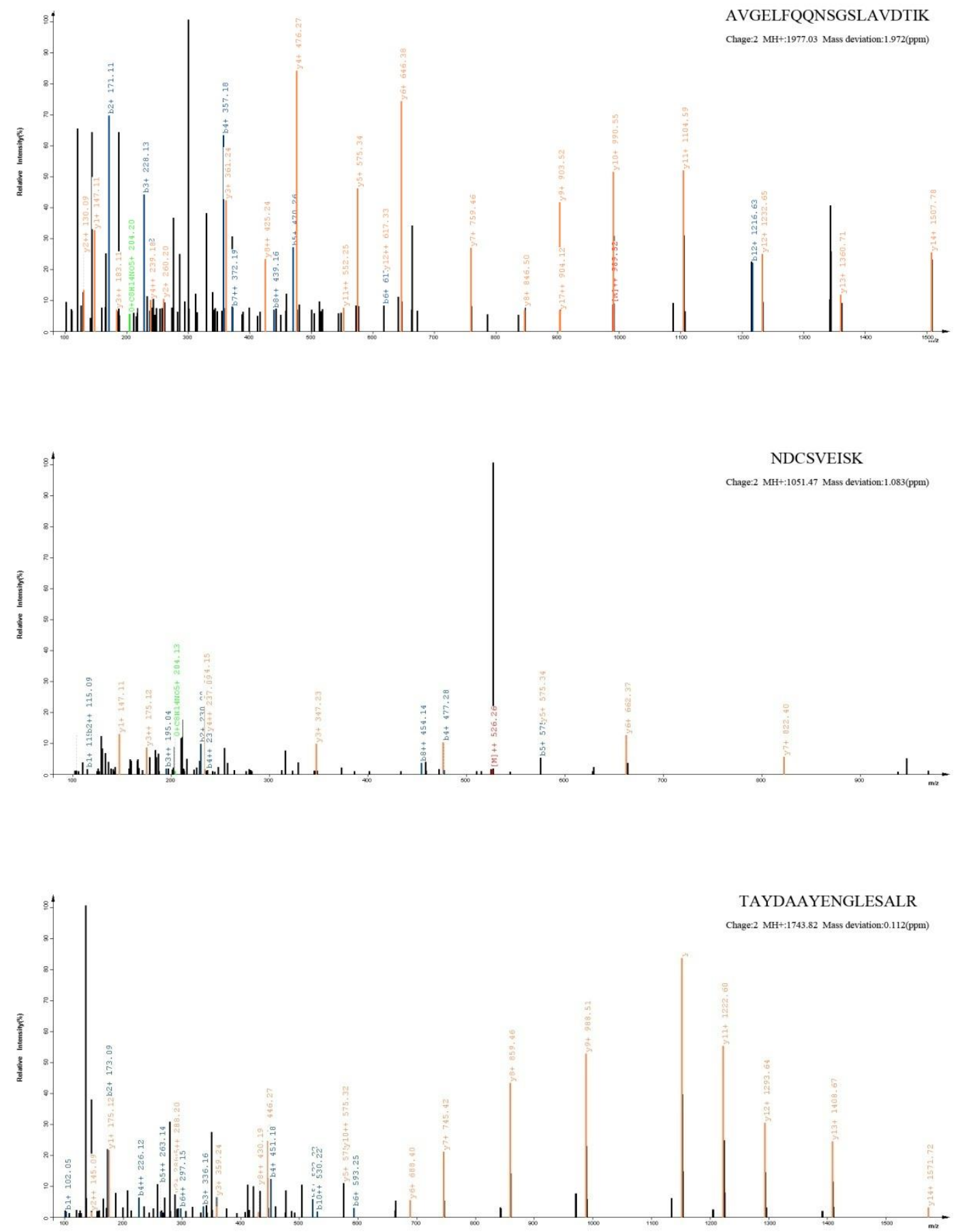

ANX-like 2 

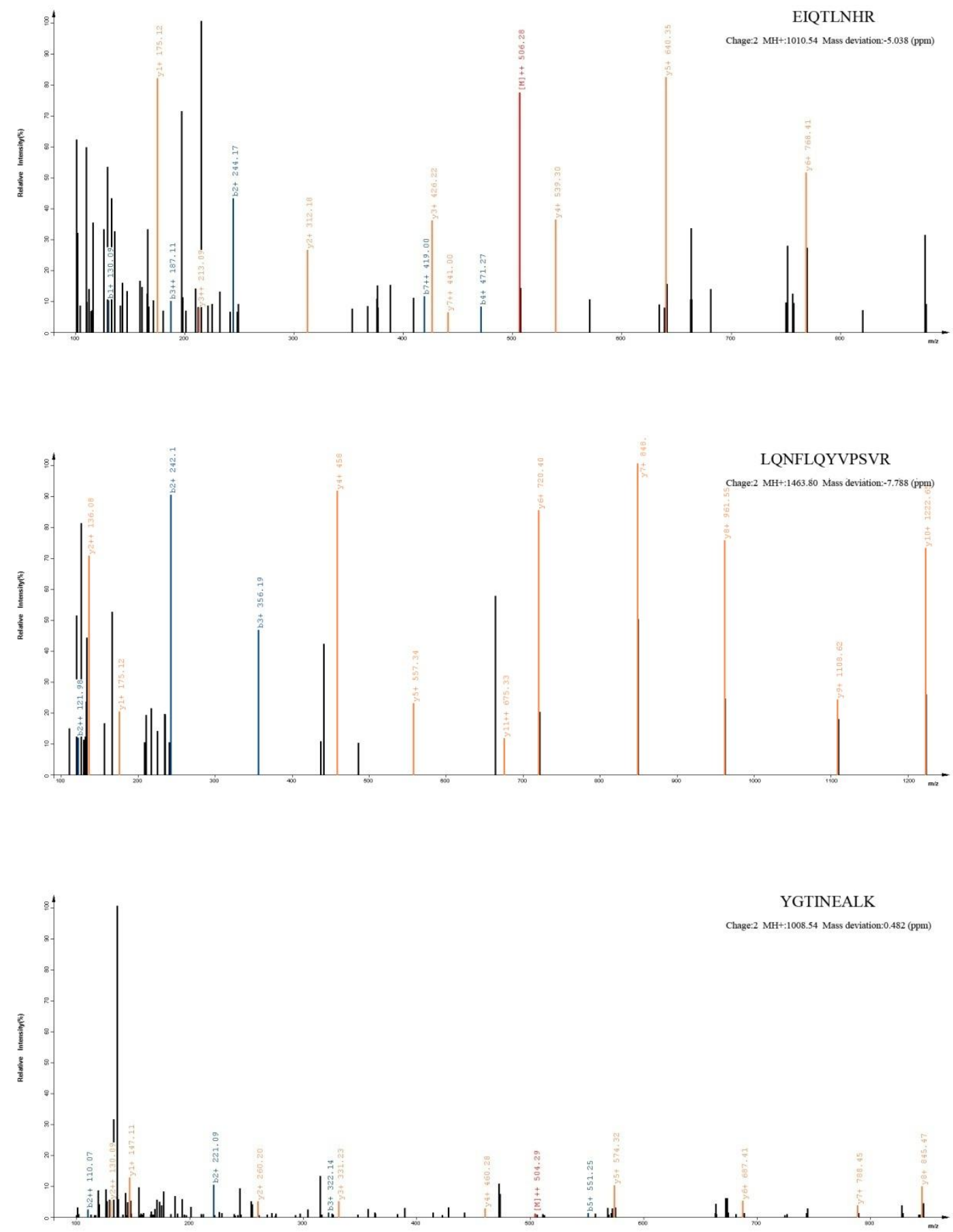

carbonic anhydrase (CA) 

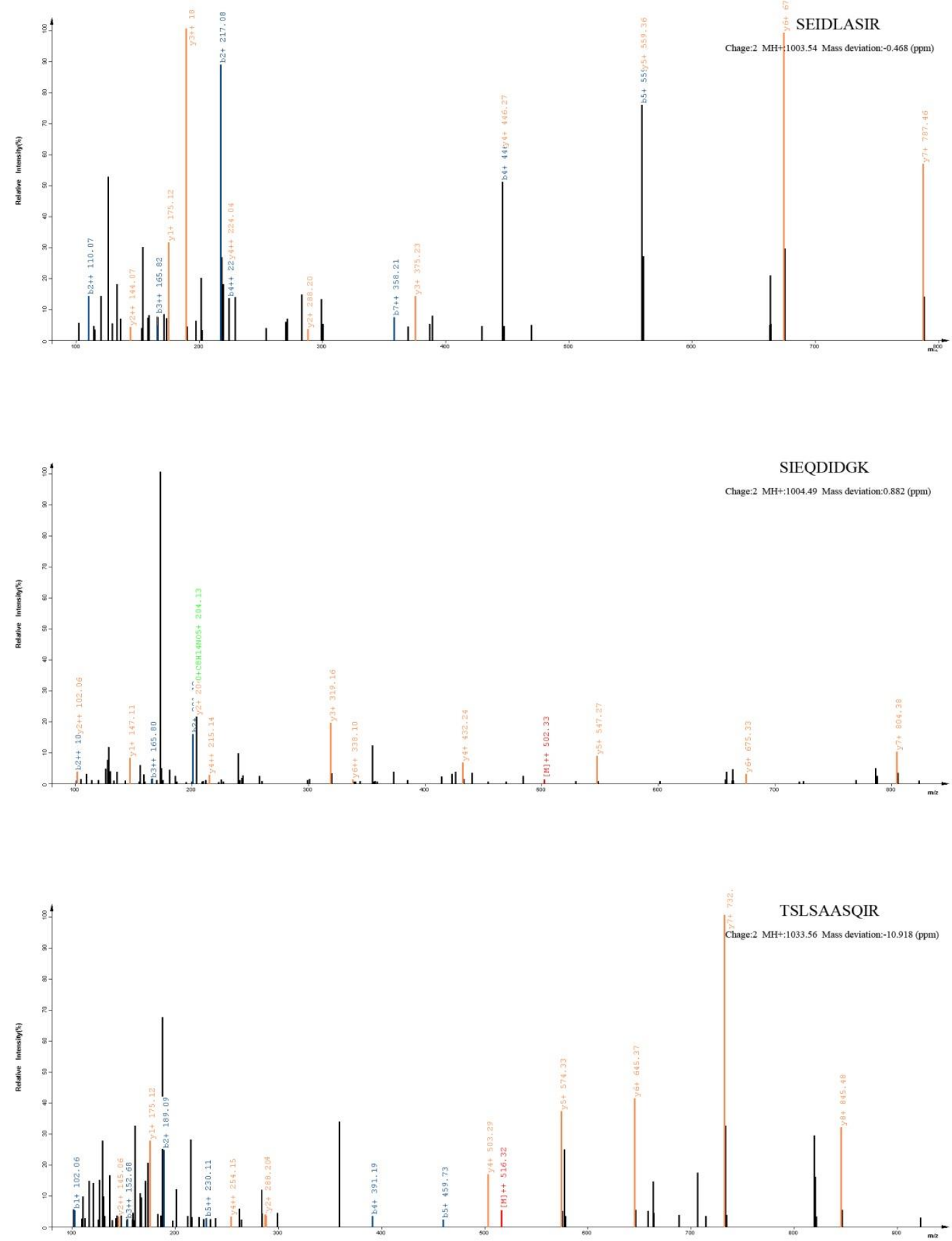

ANX-like-5 
Annotated tandem mass spectra of five gelling saliva proteins of $N$. lugens
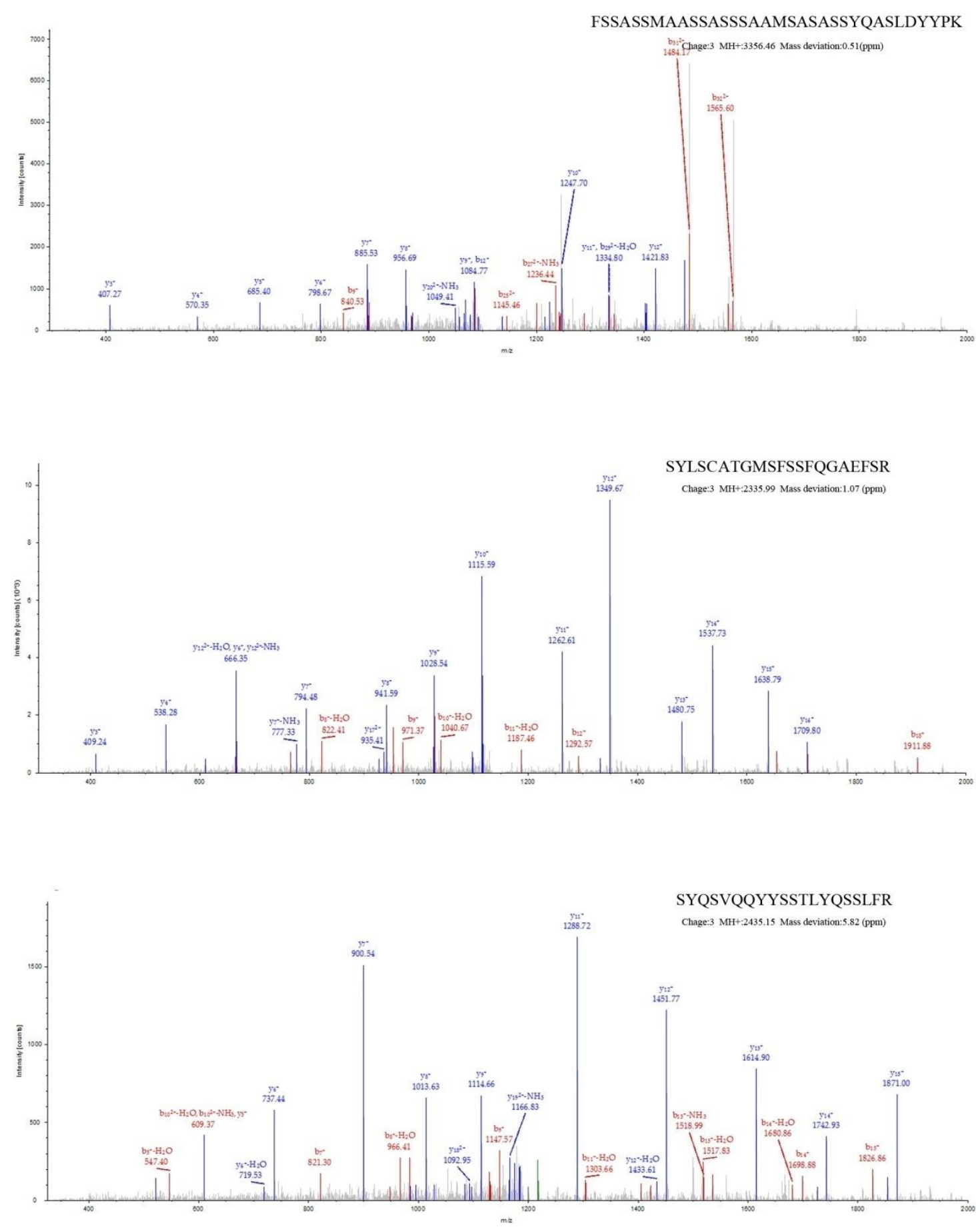

mucin-like protein 

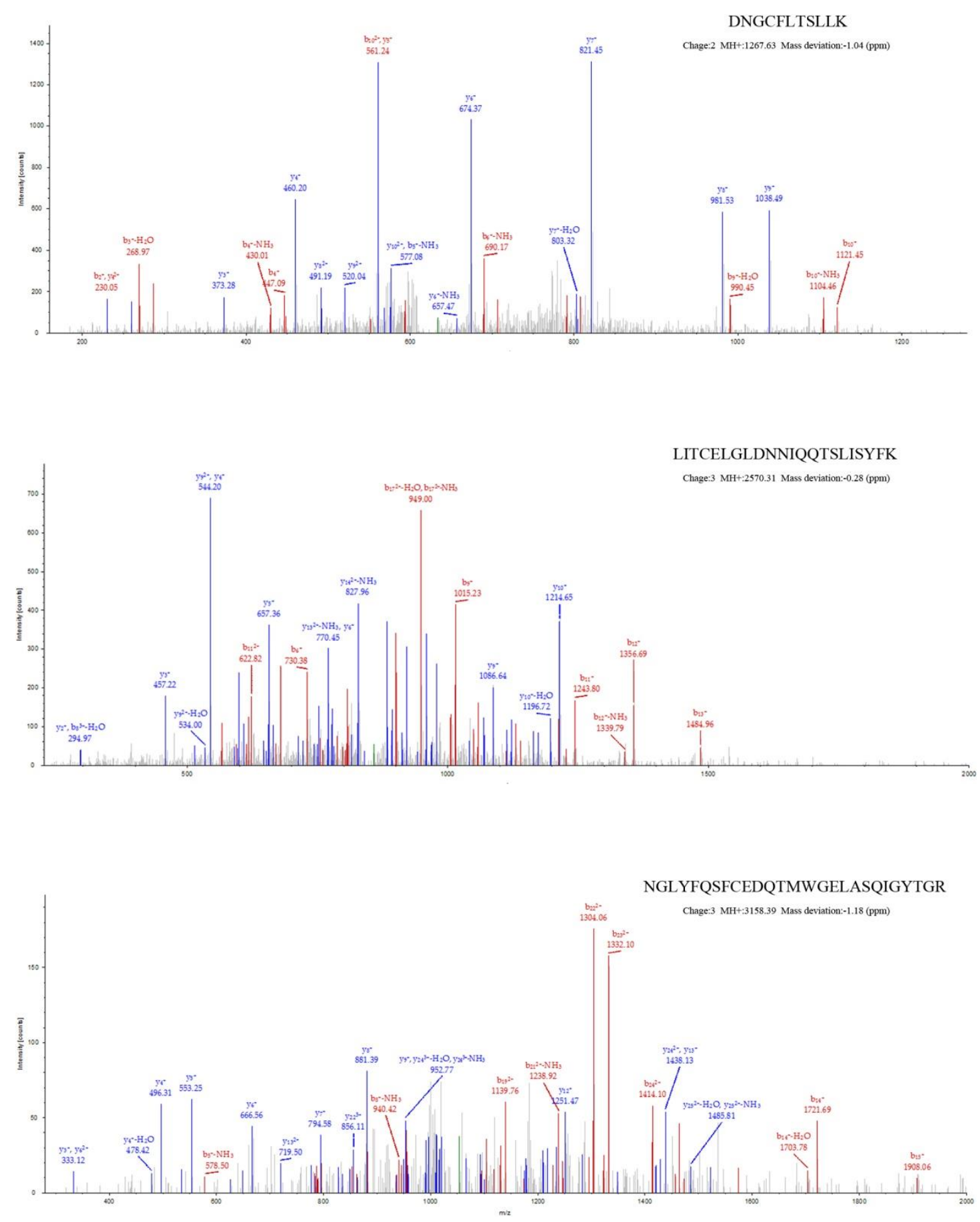

salivap-3 

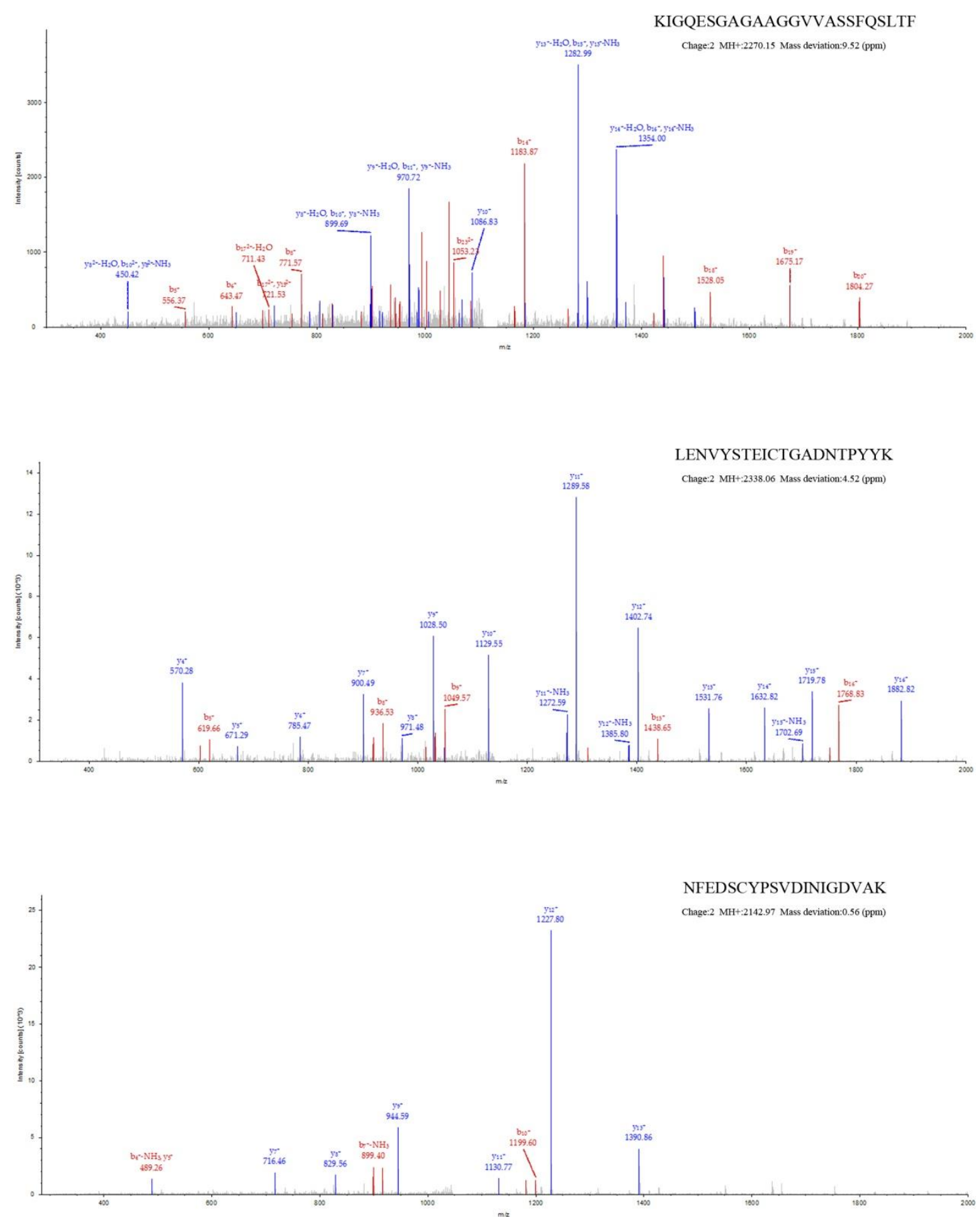

salivap-4 

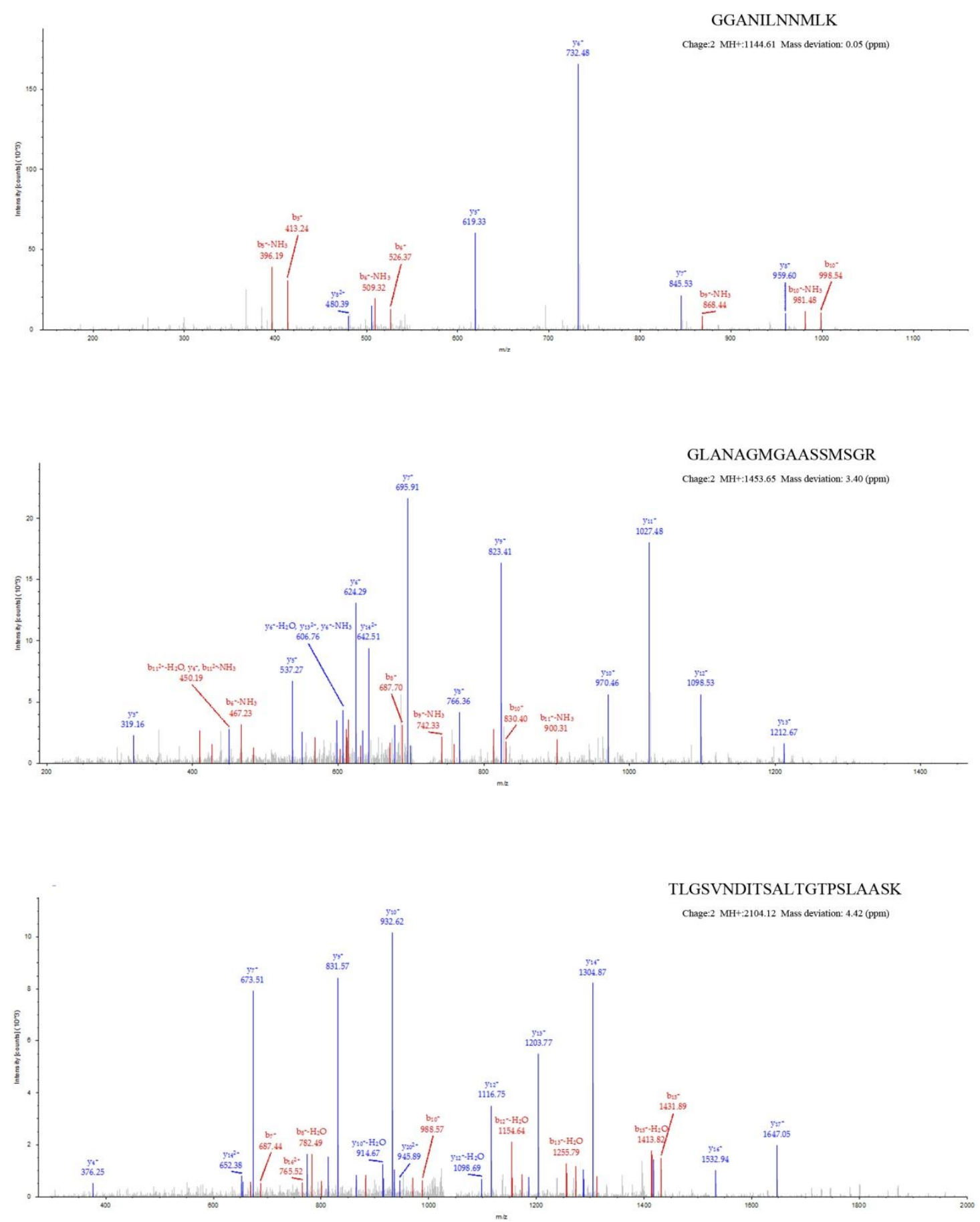

salivap-5 

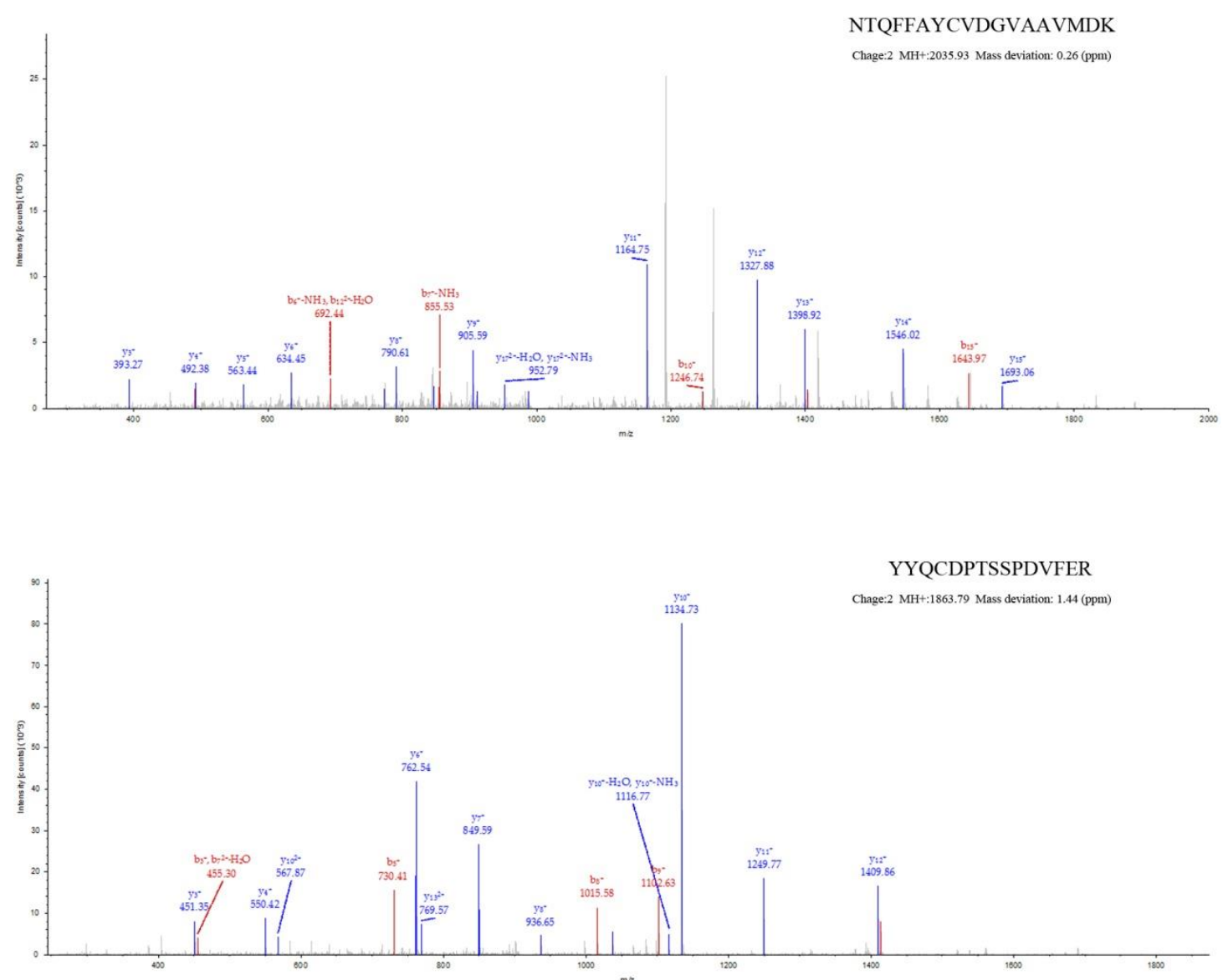

peritrophin-like protein-1

Figure S3. Annotated tandem mass spectra of five watery and five gelling saliva proteins. The precursor $\mathrm{m} / \mathrm{z}$, charge state and mass deviation are shown in each spectrum. 
Table S1. Primers used in quantitative real-time PCR and double stranded RNA synthesis

\begin{tabular}{|c|c|c|}
\hline Genes & Forward primer $\left(5^{\prime}-3^{\prime}\right)$ & Reverse primer (5'-3') \\
\hline Nl18S rRNA & CGCTACTACCGATTGAA & GGAAACCTTGTTACGACTT \\
\hline ANX-like 2 & CTCAACAGGGCCATCCAT & TGCTGCTCCTATCTCCAA \\
\hline ANX-like 3 & GGACCCTTTCAAAACCTTT & TTCGGATATCAGCCATATC \\
\hline ANX-like 4 & ATCAGGCAAGAATATATCC & GTAAGTCCAATCATCAGTAC \\
\hline Salivap-2 & CCCAAACCCTATCACTACCG & CTCTGGACAACTGGCGAAAT \\
\hline Salivap-3 & ACTACCACAACCACCACCACT & TCCTTATCATCGCTGTATCCC \\
\hline Salivap-4 & TCTGCCATCTTCCATATCACT & GATGACAGAACGACGAAAGTA \\
\hline Salivap-5 & AGCAGCAGGTGATAAGATTG & AACTACGGGTGGAGTGAGGG \\
\hline Salivap-6 & TCCAGGCGTCTGTATCCTAC & TTCAGGTTATCCAGGTAGCA \\
\hline VDDP-4 like & GTCAAAGAGCACTTACCACCG & GTTCCGCAAATATTGCACGAA \\
\hline GGT1 & TGGAGACGCCGTTTCTGTTA & $\begin{array}{l}\text { GTTGGCTGGTGTCGGAGGTA } \\
\end{array}$ \\
\hline PLCXD1 & ACTTATGATGGCCTTTGGATC & CAGCAGTGTAGCCGAGGTATG \\
\hline$C A$ & AACAACGCCACCCTTTAC & CTGGACACGTCCCTTCAC \\
\hline$L C A T$ & ACACCAGCAAAGTATTCACCG & CTGCCCAACAGTCTACAAAGG \\
\hline$P K L$ & $\begin{array}{l}\text { TTGGTATCGTTTCTTACGGTGAT } \\
\end{array}$ & TGCTCCAGTAGGTCGTGTTTC \\
\hline
\end{tabular}




\begin{tabular}{|c|c|c|}
\hline mucin like protein & TCAGGAACTTTGCCAGACGC & CGGGAAGCAGCACTCCACAT \\
\hline$L A P$ & CCGAATCGAATAATGTGCTT & TAACGCTTGAGGAGGGTAAT \\
\hline peritrophin-like protein-1 & ATGCCATCAAAGGCGAGTG & CGGGATAGGAGCAGACAGG \\
\hline peritrophin-like protein-2 & GATACAACCCTGTGGATGAG & GTCCAGGATATTCGCAAACT \\
\hline maltase 2-like & AAGCAGCCAGACTTGAACTAC & TGGTCATATTGTGAACGAGGT \\
\hline cathepsin B-like protease & ACTGCGGTTTCGGCTGTGAGG & GGTGCTATCGGGTACGGTTGG \\
\hline aminopeptidase N-like & TCCAAGCACTACCTCCACAAT & TGAAGAATACAGCACCAGCAA \\
\hline trypsin-like protease & GTGGTGCTCTGTATTGATTGTAG & GACACCTGGTAAGGAATCTCA \\
\hline vitellogenin & AGCCTCTACCAGATGACCCG & TTCCTTGCTTTGCTCCCAGT \\
\hline carboxylesterase & CTTTGCCTCAACTAACCA & TTCCTGTAAGTCGGGTCT \\
\hline GAPDH & GTCTGGTCCTTAGGGCTTCCT & CAGTTTCCTTCGGCTTTCACAT \\
\hline actin & CCCCATCTATGAAGGTTATGCC & CTCAGCGGTGGTTGTGAAGC \\
\hline alpha-GalNAc & AAGGCAGTGCTACAGAACAGA & TAGGGAAAGGAATACCATCAG \\
\hline ATP synthase & ACGAGATGATTGAGTCGGGTGT & GGTGAAGCGGAAGATGTTGTC \\
\hline RhoGAP & GACCATCACTTCGGCACTCAA & GGACACGCTCATCAGGTTCTT \\
\hline \multicolumn{3}{|c|}{ Primers used in double stranded RNA synthesis } \\
\hline GFP & TAATACGACTCACTATAGGGAGAATGAGTAAAGGAGAAGAACTTTTC & TAATACGACTCACTATAGGGAGATTTGTATAGTTCATCCATGCCATGT \\
\hline ANX-like 1 & TAATACGACTCACTATAGGGAGATTCGCTTCAAAAAGGAGGG & TAATACGACTCACTATAGGGAGAGAAAAATACTTGGCTGGGC \\
\hline ANX-like 2 & TAATACGACTCACTATAGGGAGATTGATGGAGGAAAGGACAG & TAATACGACTCACTATAGGGAGAGCGAAGTATTTGAAAGGGT \\
\hline ANX-like 3 & TAATACGACTCACTATAGGGAGAGCTGAGAAATGTTTGTGCT & TAATACGACTCACTATAGGGAGAAAAGGTTTTGAAAGGGTCC \\
\hline ANX-like 4 & TAATACGACTCACTATAGGGAGATAGGCAAAGCAGGAAGACC & TAATACGACTCACTATAGGGAGAAACCGCCAGAAATAAATCG \\
\hline ANX-like 5 & TAATACGACTCACTATAGGGAGACCAGGTCCAGTGTCGTCAT & TAATACGACTCACTATAGGGAGAGGCCATCCACATCTTTCATA \\
\hline Salivap-2 & TAATACGACTCACTATAGGGATGAAGGGAATCGTACTGTGTG & TAATACGACTCACTATAGGGTACTGGGTGTAGTGGTGATCAG \\
\hline Salivap-3 & TAATACGACTCACTATAGGGAAGGAGAGAAAGATCCACAG & TAATACGACTCACTATAGGGGCATTTTCCACATTTTCCTCCG \\
\hline Salivap-5 & TAATACGACTCACTATAGGGGATGCAGGACTGGGAATGGTG & TAATACGACTCACTATAGGGTCAAACTACGGGTGGAGTGAG \\
\hline
\end{tabular}




\begin{tabular}{|c|c|c|}
\hline Salivap-7 & TAATACGACTCACTATAGGGATGAGGGCTGCCCTGATTCTTC & TAATACGACTCACTATAGGGCTAGTAGACAACCTGTGGTCC \\
\hline Salivap-8 & TAATACGACTCACTATAGGGCGCAGCAGCTGACTCCTTTGC & TAATACGACTCACTATAGGGATGGGAATCCTGGGTATGGG \\
\hline mucin-like protein & TAATACGACTCACTATAGGGTCTGTGGTGTTCAATCCTGGG & TAATACGACTCACTATAGGGTTGGAATTCTGGTCCACGAAC \\
\hline$V D D P$ & TAATACGACTCACTATAGGGAGAGACAAACGTGAACAGCCT & TAATACGACTCACTATAGGGAGATATCTCCTCTCCAAGCGC \\
\hline VDDP-4 like & TAATACGACTCACTATAGGGAGAGATTCAGTCACCATTTCACCCA & TAATACGACTCACTATAGGGAGAATAAACCCAGTCGCCAATACCA \\
\hline GGT1 & TAATACGACTCACTATAGGGAGAGTCCCTTACATTCCCCATCCCCATT & TAATACGACTCACTATAGGGAGACACTCCAGGTCACTTTACCGCTTCC \\
\hline PLCXD1 & TAATACGACTCACTATAGGGAGAGCAAACATACCTCGGCTAC & TAATACGACTCACTATAGGGAGACATCTTCTCGCTTCACTCG \\
\hline$C A$ & TAATACGACTCACTATAGGGAGATGATGGCATTGTCGTTGAGATA & TAATACGACTCACTATAGGGAGATGTAAAGGGTGGCGTTGTTAGA \\
\hline$L C A T$ & TAATACGACTCACTATAGGGAGACCAGCAAAGTATTCACCG & TAATACGACTCACTATAGGGAGAACCCGTTTCTTGCCATTA \\
\hline$P K L$ & TAATACGACTCACTATAGGGAGAATCTTTGGATTCGGTTCA & TAATACGACTCACTATAGGGAGATCAGGAGGTGGCTTGTAG \\
\hline$L A P$ & TAATACGACTCACTATAGGGAGATACCCTGGGAGCAGAGTAGTTC & TAATACGACTCACTATAGGGAGAAGACGCCTCACATCTTTCATC \\
\hline
\end{tabular}

